\title{
Echo-Doppler avec imagerie couleur des artères caverneuses
}

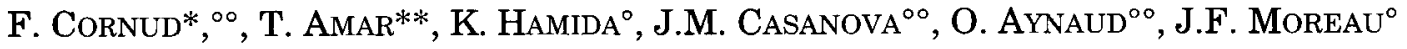 \\ * Clinique radiologique, 15 avenue Robert Schuman 75007, Paris ; * Uro-Andrologue, 19 \\ Avenue Victor Hugo, 75016, Paris ; ${ }^{\circ}$ Hopital Necker, 149 Rue de Sèvres, 75015, Paris. ; ${ }^{\circ 0}$ \\ Collège Européen et Francophone d' Urologie Libérale, 10 Rue César Franck, 75015, Paris
}

\section{RESUME}

L'étude en Doppler pulsé permet un enregistrement sélectif des artères caverneuses. Introduit en 1985 , son intérêt a été renforcé par l'utilisation de l'imagerie couleur en 1989 qui permet de localiser bien plus facilement l'artère caverneuse et par conséquent une mesure plus précise des vitesses circulatoires. Néanmoins, plusieurs controverses persistent sur le seuil du pic systolique admis $(25 \mathrm{~cm} / \mathrm{s})$ pour différencier les patients atteints d'insuffisance artérielle des patients normaux. D'autres paramètres Doppler, comme le temps d'ascension systolique ou le calcul de l'accélération, doivent être utilisés. D'autre part, il est également apparu que les vitesses diastoliques élevées en fin d'examen ne signifiaient pas toujours incompétence veinocaverneuse. Nous présentons dans cette revue la technique, les résultas normaux et anormaux du Doppler pénien avec imagerie couleur, ses limites et redéfinissons sa place en tenant compte de l'efficacité croissante du traitement par les injections intracaverneuses

Mots clés : Artères caverneuses, écho-Doppler couleur, imagerie, injections intracaverneuses.

\section{INTRODUCTION}

L'étude par écho-doppler des artères caverneuses a longtemps reposé sur l'enregistrement des artères péniennes par Doppler continu couplé à la mesure de l'index pénobrachial. Cet examen peu sensible et peu spécifique [16] a été progressivement remplacé par l'étude en Doppler pulsé qui permet un enregistrement sélectif des artères caverneuses. Introduit en 1985 par Lue et Col. [11] , son intérêt a été renforcé par l'utilisation de l'imagerie couleur en 1989 [2, 15] qui permet de localiser bien plus facilement l'artère caverneuse et par conséquent une mesure plus précise des vitesses circulatoires. Néanmoins, plusieurs controverses persistent sur le seuil du pic systolique admis $(25 \mathrm{~cm} / \mathrm{s})[13$, 18] pour différencier les patients atteints d'insuffisance artérielle des patients normaux. D'autre part, il est également apparu que les vitesses diastoliques élevées en fin d'examen ne signifiaient pas toujours une incompétence veino-caverneuse [15]. L'écho-doppler avec imagerie couleur a donc des limites qui vont être exposées dans le chapitre qui suit.

\section{TECHNIQUE}

Il est toujours précédé d'une injection intracaverneuse d'une drogue vaso-active (Figure 1a). Le chlorhydrate de Papavérine a été remplacé par une injection de 10 microgrammes de Prostaglandine E1, substance 


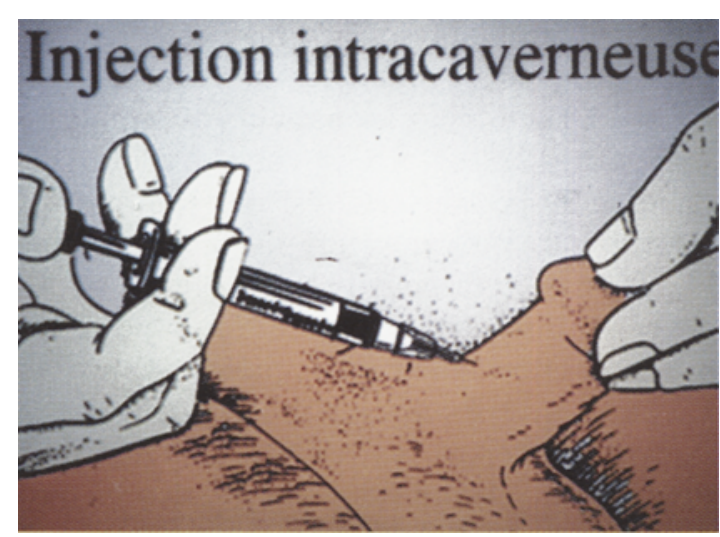

Figure 1a : Injection intracaverneuse. Elle est faite au moyen d'une aiguille de $25 \mathrm{G}$ longue d' un cm montée sur une seringue à insuline. Aucune résistance ne doit être ressentie pendant l'injection. Une résistance excessive signifie que l'on est dans les tissus sous cutanés, ou que l'on a transfixié l'albuginée vers le spongieux.

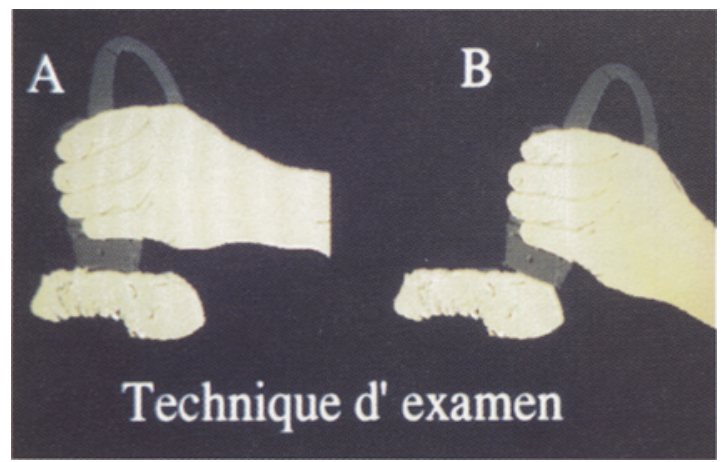

Figure 1b: La verge est repliée sur le ventre. Les artères sont repérées en mode couleur d̀ la partie moyenne de la verge (A) puis sont suivies jusqu'à la racine (B) où les conditions de recueil sont les plus favorables en mode Doppler.

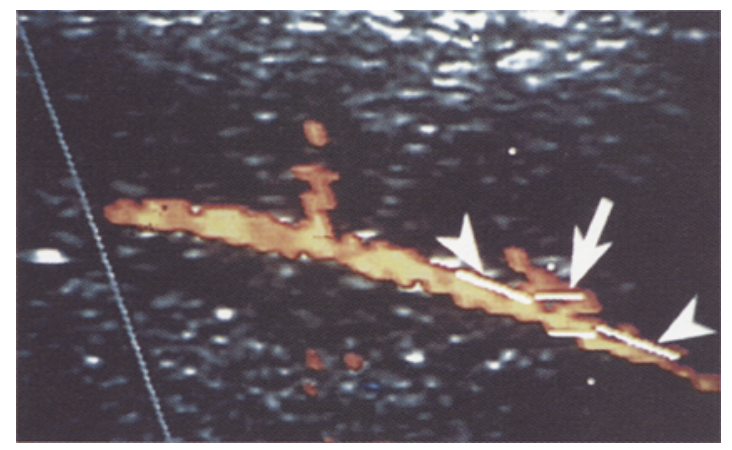

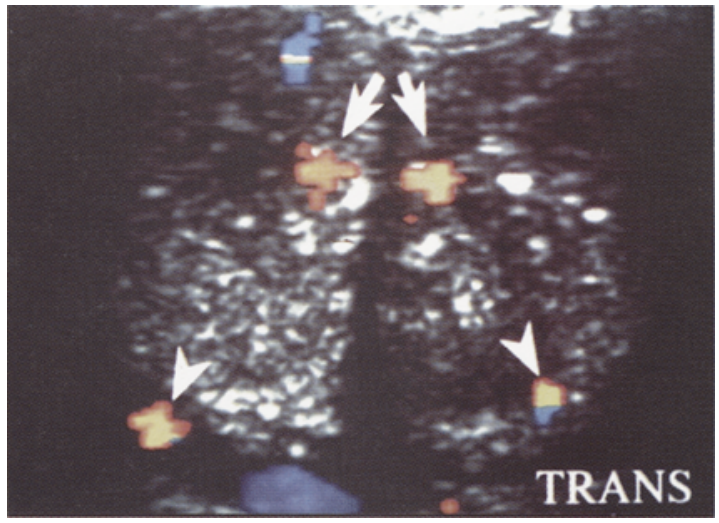

Figure 2 : Anatomie vasculaire en Doppler couleur

2a : Anatomie couleur normale, montrant les deux artères caverneuses (flèche) cheminant au centre des corps caverneux. Lesdeux artères dorsales profondes (têtes de flèche) et la veine dorsale profonde (médiane et codée en bleu) sont bien visibles.

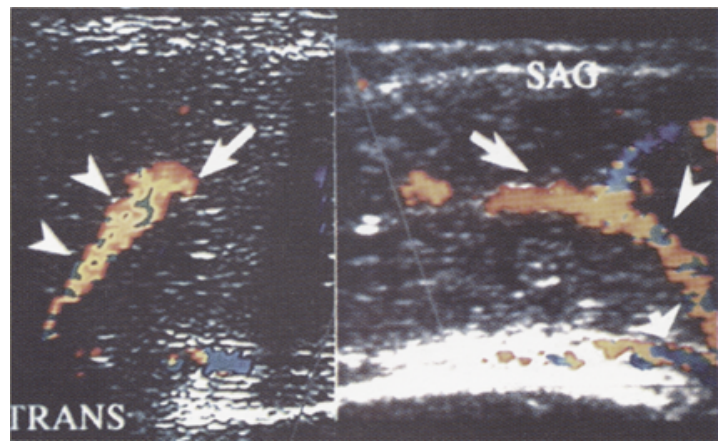

2b:Communication dorso-caverneuse, coupe transversale montrant l'artère caverneuse au centre du corps caverneux (flèche) donnant une volumineuse collatérale (tête de flèche) rejoignant l'artère dorsale profonde. Cette variation anatomique doit être repérée pour déterminer le siège d'enregistrement des vitesses circulatoires.

$<-$

Figure 1c: Enregistrement Doppler, le curseur Doppler (flèche) est placé dans l'artère caverneuse. La correction d'angle est alors appliquée pour faire coüncider l'axe du curseur correcteur (tête de flèche) et l'axe de l'artère. 
désormais approuvée par l'A.M.M. pour cet usage. L'écho-Doppler est réalisé par la plupart des auteurs sur la face ventrale de la verge (Figure 1b). Celle-ci est repliée sur le ventre, le patient la maintenant en posant la pulpe d'un index sur le gland. Il n'est pas utile de procéder à un enregistrement à l'état flacide car le flux dans les artères y est soit absent, soit très intermittent. Il n'est pas non plus utile de mesurer le calibre des artères caverneuses qui est très variable d'un individu à l'autre et qui est de plus difficile à calculer de façon reproductible avec une sonde de 7,5 Mhz, fréquence la plus souvent utilisée pour l'examen. Après l'injection, le curseur Doppler est placé dans l'artère caverneuse (Figure 1c) repérée par l'imagerie couleur. La correction d'angle est alors appliquée pour faire coïncider l'axe du curseur correcteur et l'axe de l'artère. L'enregistrement est fait sagittalement à la base de la verge (Figure 1b). Ce lieu d'enregistrement doit être pris comme référence, car les vitesses systoliques baissent d'environ $50 \%$ de la base de la verge jusqu'à la partie moyenne du pénis [9].

\section{RESULTATS}

\section{Imagerie couleur (Figure 2)}

Pendant les dix minutes suivant l'injection, un balayage couleur est réalisé à la fois transversalement et sagittalement pour étudier l'anatomie normale des artères (Figure 2a) ou détecter une variante anatomique. La plus fréquemment observée est une communication entre l'artère dorsale profonde et l'artère caverneuse (Figure $2 b$ ) ; cette dernière peut d'ailleurs naître de l'artère dorsale un peu en aval de la base de la verge. La variation est à connaître pour déterminer le lieu d'enregistrement des vitesses circulatoires.

\section{Analyse spectrale}

\section{a) Les vitesses systoliques,(Figure 3)}

Des vitesses systoliques inférieures à $25 \mathrm{~cm} / \mathrm{s}$ traduisent une insuffisance d'apport artériel.
Les corrélations avec l'artériographie [1] ont montré que le pic systolique moyen était inférieur à $10 \mathrm{~cm} / \mathrm{s}$ chez les patients atteints d'insuffisance artérielle sévère. Au delà de $35 \mathrm{~cm} / \mathrm{s}$, l'apport artériel est en règle normal avec une valeur moyenne du pic systolique supérieure à $45 \mathrm{~cm} / \mathrm{s}$ [1]. En revanche, les corrélations sont moins bonnes pour les vitesses intermédiaires comprises entre 25 et $35 \mathrm{~cm} / \mathrm{s}$, car il existe un chevauchement important entre les apports normaux et insuffisants. Dans ces cas difficiles, il faut accorder de la valeur à une asymétrie des vitesses circulatoires entre l'artère droite et l'artère gauche. Une différence de plus de $\mathbf{1 0}$ $\mathrm{cm} / \mathrm{s}[2,7]$ constitue un argument pour une insuffisance d'apport. D'autres paramètres Doppler permettent également de mieux apprécier l'apport artériel. Le premier est le temps de montée systolique (Figure 3a), calculé depuis le pied de la systole jusqu'à l'inflexion de la courbe du pic de montée. Un temps d'ascension supérieur à 110 millisesondes [14] représente probablement le meilleur signe d'insuffisance d'apport, mais sa sensibilité est faible, ne dépassant pas $30 \%$ dans notre expérience [3], contrairement aux résultats d'autres études [13]. L'avantage de ce signe est qu'il est indépendant de la correction d'angle [13]; il est donc simple à utiliser, mais une valeur normale n'élimine en rien une artérite. L'autre paramètre Doppler est la mesure de l'accélération. C'est le rapport entre la différence des vitesses systoliques et diastoliques sur le temps d'ascension systolique (Figure $3 \mathrm{~b}$ ). La normale est de $4 \mathrm{~m} / \mathrm{s}^{2}$ (18). L'accélération a une sensibilité de $100 \%$ au détriment d'une spécificité modeste $(46 \%)$ pour détecter une insuffisance d'apport. La mesure de l'accélération est cependant le seul moyen de la suspecter quand les vitesses systoliques sont comprises entre 25 et $35 \mathrm{~cm} / \mathrm{s}$ avec un temps d'ascension normal (Figure 3b). Le calcul de l'accélération est moins simple que celui du temps $d^{\prime}$ ascension car elle doit être mesurée en faisant une correction d'angle Doppler, comme pour les vitesses circulatoires. 


\section{b) Vitesses diastoliques (Figure 4)}

Les vitesses télédiastoliques sont corrélées à la pression intra caverneuse [17]. Elles ne sont interprétables que si les vitesses systoliques sont normales, c'est à dire supérieures à $35 \mathrm{~cm} / \mathrm{s}$. En dessous de $40 \mathrm{~mm} \mathrm{Hg}$ (Figure $4 a$ ), les vitesses diastoliques sont supérieures à $5 \mathrm{~cm} / \mathrm{s}$, ce qui correspond cliniquement à l'intumescence. Quand les vitesses systoliques sont comprises entre 40 et $60 \mathrm{~mm} \mathrm{Hg}$ les vitesses diastoliques chutent en dessous de $5 \mathrm{~cm} / \mathrm{s}$ (Figure $4 \mathrm{~b}$ ) et s'annulent lorsque la pression atteint 60 $\mathrm{mm} \mathrm{Hg}$ (Figure 4c). Cliniquement, la rigidité n'est pas constante dans cette tranche de valeurs [3] ; la chute des vitesses diastoliques en dessous de $5 \mathrm{~cm} / \mathrm{s}$ signifie cependant que l'occlusion veineuse se produit normalement. A $80 \mathrm{~mm}$ de mercure, les vitesses diastoliques s'inversent (Figure $4 \mathrm{~d}$ ), donnant le classique reflux proto-diastolique, phase qui correspond à une rigidité ou à une érection.

La corrélation entre les vitesses diastoliques et le flux dans la veine dorsale profonde, suggérée par Fitzegerald et coll. [7] n'est pas bonne ; un flux veineux persistant peut être enregistré même avec des vitesses diastoliques nulles [3], voire négatives (Figure 4e). Ce flux correspond au sang veineux du gland et du corps spongieux dont les veines de drainage rejoignent, comme les veines émisssaires, la veine dorsale profonde. Il peut donc y être enregistré, même si les veines émissaires sont totalement comprimées contre l'albuginée par l'expansion des sinusoides [12].

c) Signification d'une vitesse télédiastolique élevée en fin d'examen (Figure 5)

Elle signifie que le système veineux occlusif n'a pas fonctionné au moment de l'examen.(Figure 5a). Elle n'est interprétable que si les vitesses systoliques sont normales, c'est-à-dire supérieures à $35 \mathrm{~cm} / \mathrm{s}$. Une vitesse télédiastolique élevée ne signifie pas en revanche qu'existe une insuffi- sance veino-caverneuse [15]. Celle-ci doit être confirmée, si nécessaire, par une cavernométrie avec cavernographie réalisée au débit de maintien de l'érection (Figure 5b). Au préalable, on peut proposer, au prix d'une prolongation notable du temps de l'examen, une ré-injection intracaverneuse [4], éventuellement en combinant les drogues ou une stimulation visuelle ou une auto stimulation pour déclencher l'occlusion veineuse [10]. Le Doppler couleur a cependant clarifié les indications de la cavernométrie : celle-ci n'a plus d'indications quand les vitesses diastoliques sont en dessous de $5 \mathrm{~cm} / \mathrm{s}$ car, à ces valeurs, le système veineux occlusif fonctionne normalement [3, 15]. Si une discordance était observée, il faut évoquer, comme l'a souligné Lue et coll. [10], un faux positif de la cavernométrie. Il n'est à cet égard pas rare d'observer, surtout en cas de débit de maintien compris entre 25 et $60 \mathrm{ml} / \mathrm{mn}$, une disparition complète de la fuite en répétant la mesure avec du produit de contraste dilué.

d) Enregistrement des vitesses circulatoires dans le temps

Les vitesses circulatoires doivent être mesurées 10,20 , et $30 \mathrm{mn}$ après l'injection intracaverneuse $[7,8]$. Si l'on utilise de la Prostaglandine, le pic systolique atteint sa valeur normale entre 10 et $20 \mathrm{mn}$ après l'injection dans $90 \%$ des cas environ, et entre 20 et $30 \mathrm{mn}$ après l'injection dans $10 \%$ des cas. Il en est de même pour les vitesses télédiastoliques qui ne peuvent chuter en dessous de $5 \mathrm{~cm} / \mathrm{s}$ qu'entre 20 et $30 \mathrm{mn}$ après l'injection [3].

e) Place de l'écho-doppler pénien dans l'exploration de l'impuissance suspectée d'être d'origine vasculaire

Compte tenu des progrès réalisés par les injections intracaverneuses et de l'efficacité de la Prostaglandine E1, les indications de l'écho-doppler pénien ont beaucoup baissé. Celui-ci n'est en effet utile qu'en cas de réponse non optimale à l'injection intraca- 


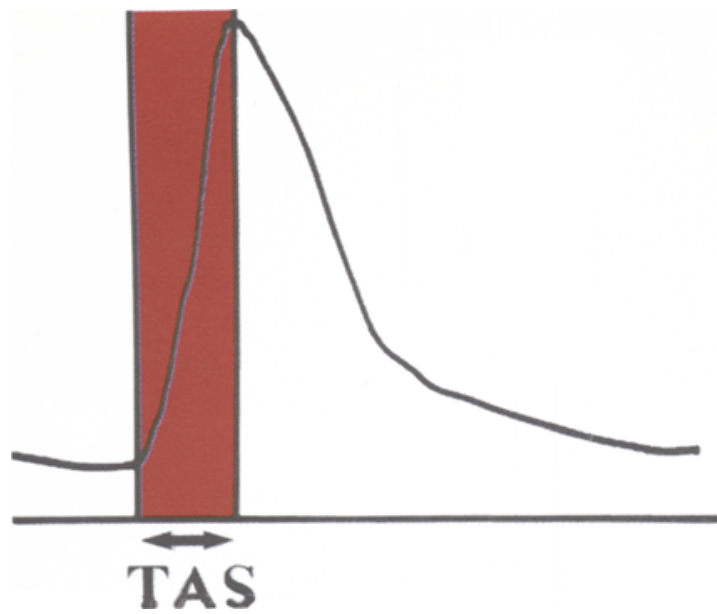

Figure 3 : Insuffisance artérielle.

3a : Schéma montrant le principe de calcul du temps d'ascension systolique (TAS).

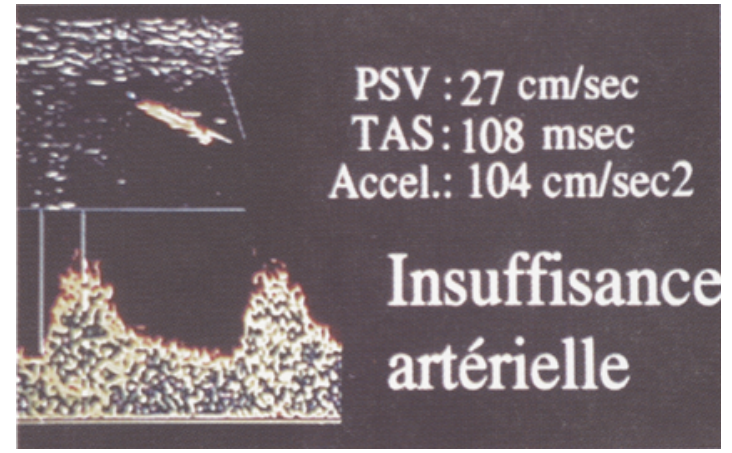

$3 b$ : Calcul de l'accélération et insuffisance artérielle

(A) : Le pic systolique (PSV) est à la limite inférieure de la normale, à $27 \mathrm{~cm} / \mathrm{s}$. Le temps d'ascension est normal (108 millisecondes) mais l'accélération est effondrée devant faire rechercher une insuffisance artérielle.

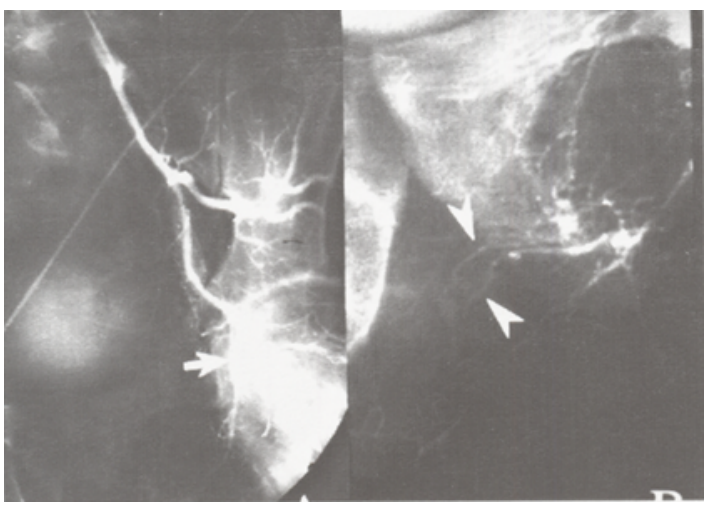

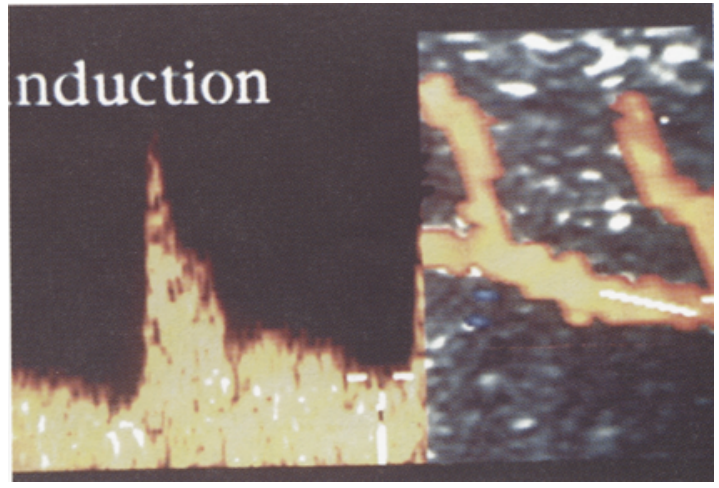

Figure 4 : Vitesses diastoliques.

4a: Lors de l'induction de l'érection, on assiste à une augmentation importante des vitesses systoliques et diastoliques.

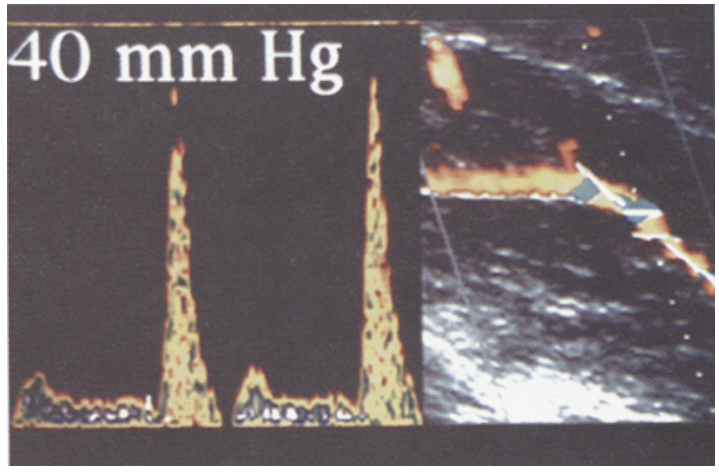

$4 b$ : Dès que la pression intra caverneuse atteint $40 \mathrm{~mm}$ de mercure, on assiste à une chute des vitesses diastoliques en dessous de $5 \mathrm{~cm} / \mathrm{s}$, alors que les vitesses systoliques restent stables.

$<-$

(B) : Artériographie honteuse. Injection sélective dans le trone antérieur de l'hypogastrique, montrant l'absence de lésion proximale. La portion proximale de l'artère $(A)$ est normale. Les lésions distales $(B)$ sont au contraire évidentes ; les artères caverneuses sont atteintes de façon diffuse avec occlusion du tronc principal et opacification de plusieurs petites branches collatérales ayant un aspect grêle (têtes de flèche). 


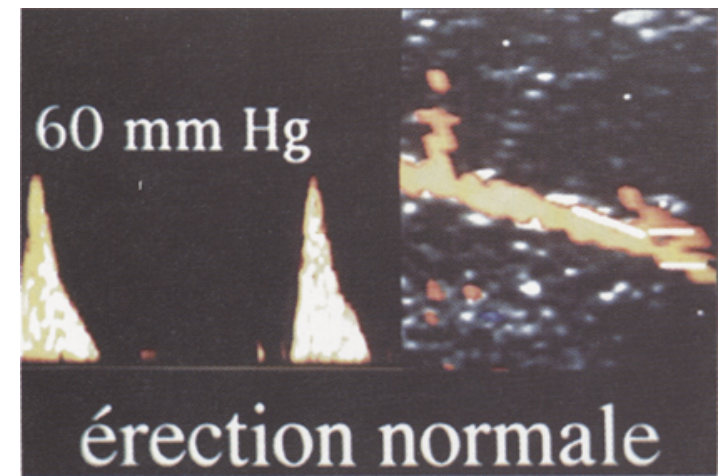

$4 c$ : Phase d'état. A partir de $60 \mathrm{~mm}$ de mercure, les vitesses distoliques s'annulent.

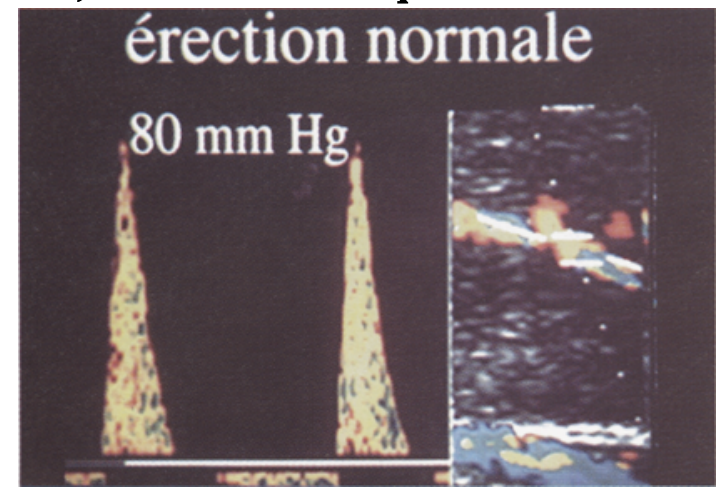

4d: Elles s'inversent lorsque la pression atteint $80 \mathrm{~mm}$ de mercure, responsables du classique reflux diastolique traduisant une érection normale.

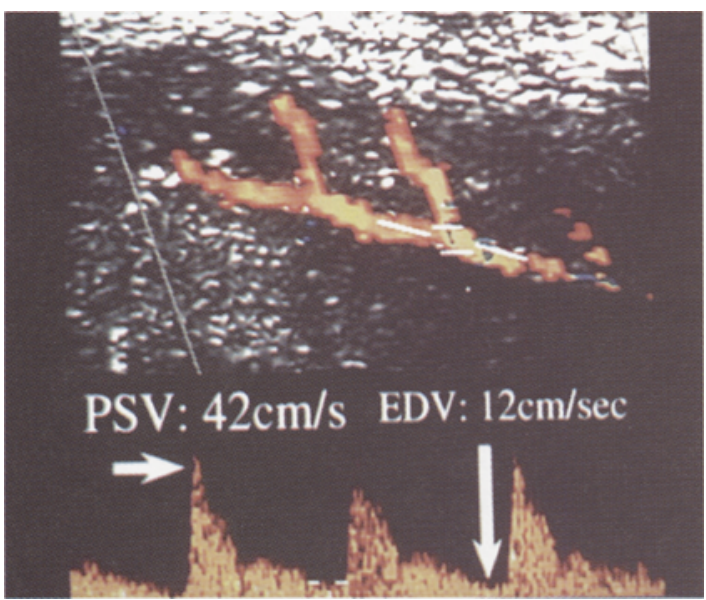

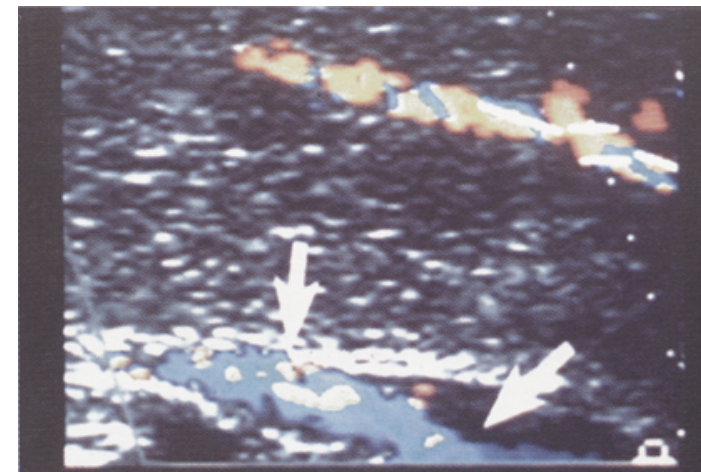

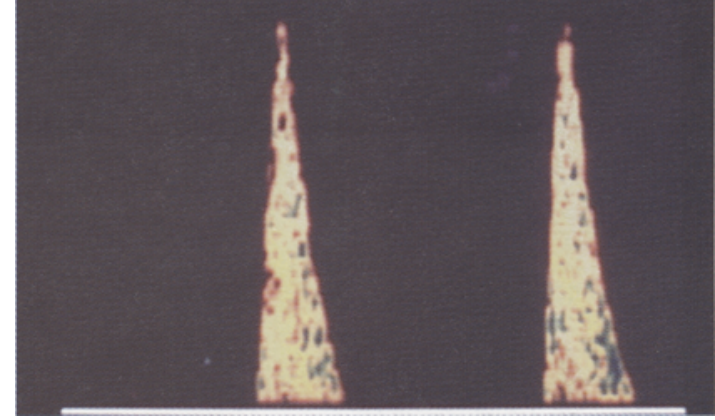

4e : Flux dans la veine dorsale profonde et occlusion veineuse. Doppler couleur montrant la persistance d'un flux dans la veine dorsale profonde (flèches), alors que l'enregistrement artériel montre une inversion des vitesses diastoliques qui garantit une occlusion veineuse normale.

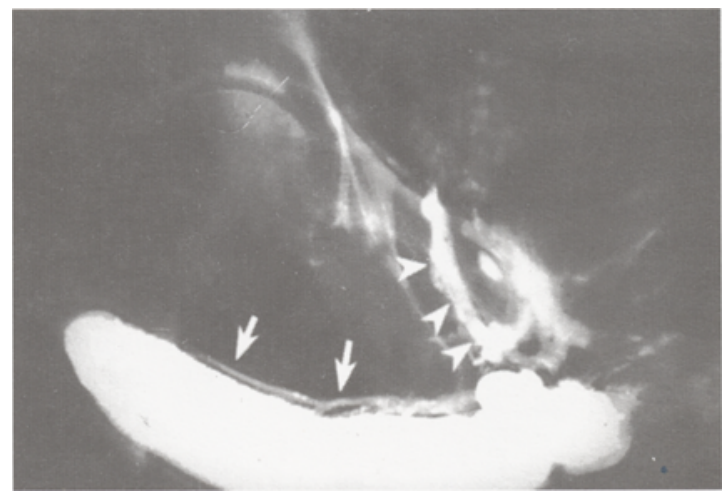

Figure 5 : Non fonctionnement du système veino-occlusif. $5 a$ : Trente minutes après l'injection, on constate la persistance d'une

vitesse diastolique élevée (EDV: $12 \mathrm{~cm} / \mathrm{s}$ ) alors que les vitesses systoliques sont stables (PSV:42 cm/s). Le système veineux occlusif n'a pas fonctionné au moment de l'examen. L'incompétence veino-caverneuse est à confirmer par une cavernométrie.

$5 b$ : Cavernographie. L'injection de contraste réalisé au débit de maintien de l'érection (75ml/mn) montre la persistance d'un drainage veineux par la veine dorsale profonde (flèche) rejoignant les plexus hypogastriques (têtes de flèche). 
verneuse, auquel cas un problème vasculaire peut être suspecté. Il persiste un doute sur l'utilisation des paramètres Doppler entre 25 et $35 \mathrm{~cm} / \mathrm{s}$. Affiner les résultats du Doppler par une artériographie dans cette situation paraît donc encore souhaitable même si le traitement n'en sera que très rarement modifié; aucune technique chirurgicale [5] ou de radiologie d'intervention [6] n'ayant fait la preuve de bons résultats à long terme dans le traitement de l'impuissance d'origine vasculaire. Le Doppler pénien utilisé avec imagerie couleur et utilisation optimale des paramètres Doppler est néanmoins devenu l'examen de référence pour tester une impuissance suspecter d'être d'origine vasculaire. Il a le mérite de prédire si le traitement proposé par l'injection intracaverneuse a des chances d'être temporaire (si le système vasculaire est normal) ou s'il risque d'être définitif ou relayé par la pose d'une prothèse pénienne dans le cas contraire.

\section{REFERENCES}

1. Benson C, Aruny J, Vickers M. : Correlation of duplex sonography with arteriography in patients with erectile dysfunction. AJR 1993 ; 160 : 71-73.

2. Benson C, Vickers M. : Sexual impotence caused by vascular disease : diagnosis with duplex sonography. AJR $1989 ; 153: 1149-1153$.

3. CORNud F. SEXUAL DISORDERS. : International uroradiology ' 96. Copenhagen.Arhus.Odense : FADL Publishers, 1996: 275-279.

4. Cornud F, Boisrond L, Bonnel D. : EchographieDoppler couleur dans l'exploration de l'impuissance d'origine vasculaire. Progrès en Urologie 1992 ; $2: 420-426$.

5. DeanKnoll L, Furlow W. : Venous and arterial surgery for vasculogenic impotence. In: Hasmat A, Das S, eds. The penis. Philadelphia. London : Lea and Febiger, 1993 : 174-190.

6. Delcour C, Bruninx G, Roger T, et al. : Explorations radiologiques de l' impuissance. Rev. Imag. Méd. 1994 ; 6 : 335-344.

7. FitzGerald S, ERickson S, Foley W, Lipchik E. : Color doppler sonography in the evaluation of erectile dysfunction : patterns of temporal response to Papaverine. AJR $1991 ; 157$ : 331-336.
8. Govier F, Asase D, Hefty T, Macclure R, PritCHETT T, WEISSMAN R. : Timing of penile color flow duplex ultrasonography using a triple drug mixture. J. Urol. $1995 ; 153: 1472-1475$.

9. Kim S, Paick J, Lee S, Chor B, Yeon K, Han M. : Doppler sonography of deep cavernosal artery of the penis : viariation of peak systolic velocity according to sampling location. J. Ultrasound Med. $1994 ; 13: 591-594$.

10. Lue T. : Erectile dysfunction : problems and challenges. J. Urol. $1993 ; 149$ : 1246-1255.

11. LUE T, HRICAK H, Marick K, Tanagho E. : Vasculogenic impotence evaluated by high-resolution ultrasonography and pulsed doppler spectrum analysis. Radiology $1985 ; 155: 777-781$.

12. Lue T, Tanagho E. : Hemodynamics of erection. In : Contemporary management of impotence and infertility. Baltimore: Williams and Wilkins, 1988. (Tanago EA LT, DaleMcLure R., ed).

13. Meuleman E, Diemont W. : Investigation of erectile dysfunction. Urol. Clin. N. Am. $1995 ; 22$ (4) : 803-819.

14. Oates C, Pickard R, Powell P, Murthy L, WhitTINGHAM A. : The use of duplex ultrasound in the assessment of arterial to the penis in vasculogenic impotence. J. Urol. $1995 ; 153: 354-357$.

15. Quam J, KIng B, JAMes E, ET AL. : Duplex and color doppler sonographic evaluation of vasculogenic impotence. AJR $1989 ; 153$ : 1141-1147.

16. SChwartz A, Lowe M, Ireton R. : A comparison of penile brachial index and angiography : evaluation of corpora cavernosa artery inflow. J. Urol. 1990 ; $143: 510-513$.

17. Schwartz A, Wang K, Mack L, Lowe M, Berger R, FELDMAN M. : Evaluation of normal erectile function with color flow doppler sonography. AJR $1989 ; 153: 1155-1160$.

18. VALJI K, BoOKSTEIN J. : Diagnosis of arteriogenic impotence : efficacy of duplex sonography as a screening tool. AJR $1993 ; 160: 65-69$.
ABSTRACT
Color Doppler sonography of the cavernosal arteries
F. Cornud, T. Amar, K. Hamida et al
Penile vascular imaging has been based for years on pharmacoangiogra- phy and pharmacocavernosometry. Doppler sonography of the cavernosal 
arteries has received considerable attention since its description in 1985. Color Doppler imaging further stimulated interest in this non invasive method, as it permitted more accurate measurement of systolic and diastolic velocities. Despite the popularity and appeal of penile sonography, the validity of accepted velocity thresholds has not been thoroughly appraised, particularly in patients with moderate alterations of systolic velocities. Other Doppler parameters, like systolic rise time and measurement of acceleration must be used in patients with subnormal systolic velocities. Moreover, it emerged that an elevated EDV was not always related to venous incompetence (venogenic impotence). In this article, based on a review of the literature and personal experience, we present the technique and results of normal and abnormal color Doppler sonography (CDS) of the cavernosal arteries and outline the limits of the technique. Its role as a screeening test is reappraised, taking into account the therapeutic efficacy of intracavernosal injections.

Key words : cavernosal arteries, color Doppler sonography, imaging, intracervosal injections. 